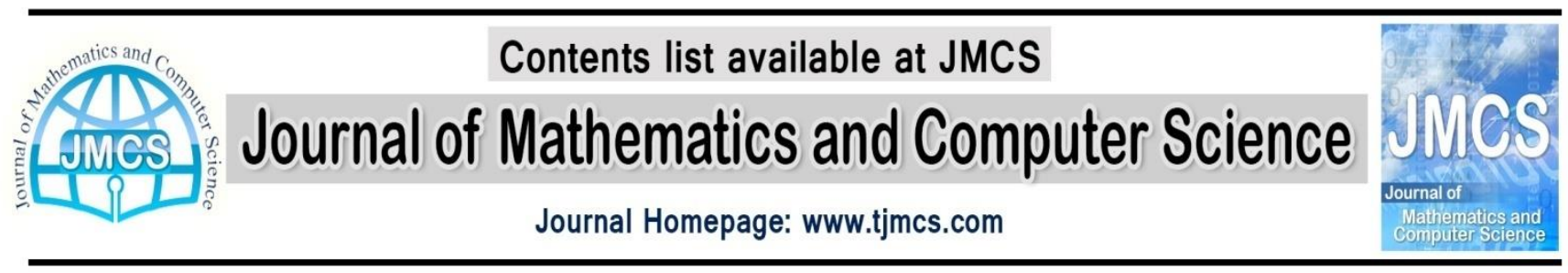

\title{
On Fuzzy Topological Spaces Involving Boolean Algebraic Structures
}

\author{
P.K. Sharma \\ Post Graduate Department of Mathematics, D.A.V. College, Jalandhar city, Punjab, India \\ pksharma@davjalandhar.com
}

Article history:

Received March 2015

Accepted July 2015

Available online July 2015

\begin{abstract}
The notion of fuzzy set was introduced by L.A. Zadeh as a generalization of the notion of classical set or crisp set. Fuzzy topological spaces were introduced by C.L. Chang and studied by many eminent authors like R. Lowen and C.K. Wong. A. Rosenfeld applied the notion of fuzzy set to algebra and introduced fuzzy subgroup of a group. Shaoquan Sun introduced the notion of fuzzy Boolean subalgebra in a Boolean algebra. In this paper, we will study fuzzy topology by involving the Boolean algebraic structure on it and introduce the notion of Boolean algebraic fuzzy topological spaces. We will examine many properties of these spaces and obtain many results.
\end{abstract}

Keywords: Fuzzy topological space (FTS), fuzzy Boolean subalgebra (FBSA), Boolean algebraic fuzzy topological space (BAFTS), fuzzy point (FP).

\section{Introduction}

Zadeh's classical paper [8] introduced the fundamental concept of fuzzy sets, upon which lies the foundation of fuzzy mathematics. The study of fuzzy topological spaces and fuzzy Boolean algebra are the two main branches of such mathematics.

General topology and Boolean algebra can be regarded as a special case of fuzzy topology and fuzzy Boolean algebra respectively, where all membership functions in question take values 0 and 1 only. Therefore, one would expect weaker results in the case of fuzzy topology and fuzzy Boolean algebra. Some basic properties of fuzzy topology are discussed in $[1,10]$ and related to fuzzy subalgebra of Boolean algebra are discussed in $[5,6]$.

In this paper, we will study the fuzzy topological spaces by involving Boolean algebraic structure on it and introduce the notion of Boolean algebraic fuzzy topological spaces. It is remarked here that Boolean algebraic fuzzy topological spaces are different from fuzzy topological Boolean algebras, as introduced by A. Parvathi and K.N. Meenakshi in [3]. 


\section{Preliminaries}

In this section, we list some basic concepts and well known results on fuzzy topology and fuzzy Boolean algebra for the sake of completeness of the topic under study. Throughout the paper, $\varnothing_{\sim}$ and $\mathrm{X}_{\sim}$ denotes the constant fuzzy sets on $\mathrm{X}$ defined by $\varnothing_{\sim}(x)=0 ; \forall x \in \mathrm{X} \quad$ and $\quad \mathrm{X}_{\sim}(x)=1 ; \forall x \in \mathrm{X}$.

Definition (2.1)[1] Let $X$ be a non-empty set and $\mathrm{I}^{\mathrm{X}}$ denotes the set of all fuzzy subsets of $\mathrm{X}$, i.e., the set of all mappings $\mu: \mathrm{X} \rightarrow \mathrm{I}$. Then a subfamily $\delta \subseteq \mathrm{I}^{\mathrm{X}}$ is said to be a fuzzy topology on $\mathrm{X}$, if it satisfies the following:

(i) $\varnothing_{\sim}, X_{\sim} \in \delta$;

(ii) If $\left\{\mu_{\mathrm{i}}: \mathrm{i} \in \Lambda\right\} \subseteq \delta$, then $\bigcup_{i \in \Lambda} \mu_{i} \in \delta$;

(iii) If $\mu, v \in \delta$ be any two members, then $\mu \cap \nu \in \delta$.

If $\delta$ is a fuzzy topology on $\mathrm{X}$, then the pair $(\mathrm{X}, \delta)$ is called fuzzy topological space (FTS). The members of $\delta$ are called $\delta$-open sets. A fuzzy set $\mu$ of $\mathrm{X}$ is said to be $\delta$-closed in $(\mathrm{X}, \delta)$ if and only if $\mu^{\mathrm{c}}$ is $\delta$-open set in $(\mathrm{X}, \delta)$. When $\delta=\mathrm{I}^{\mathrm{X}}$, then $(\mathrm{X}, \delta)$ is called a discrete fuzzy topological space and when $\delta=\left\{\varnothing_{\sim}, \mathrm{X}_{\sim}\right\}$, then $(\mathrm{X}, \delta)$ is called an indiscrete fuzzy topological space.

Remark (2.2) In the definition (2.1) if condition (i) is replaced by (i) $\forall \mathrm{r} \in[0,1]$ and $\mathrm{r}^{*} \in \mathrm{I}^{\mathrm{X}}$, defined by $\mathrm{r}^{*}(x)=\mathrm{r} ; \forall x \in \mathrm{X}$ (called constant fuzzy set in $\mathrm{X}$ ), are in $\delta$, then $\delta$ is called fully stratified fuzzy topology and the pair $(\mathrm{X}, \delta)$ is called fuzzy stratified fuzzy topological space.

Definition (2.3) [1] Let $\mathrm{X}$ and $\mathrm{Y}$ be two non-empty sets and $f: \mathrm{X} \rightarrow \mathrm{Y}$ be a mapping. Let $\mu$ and $\nu$ be fuzzy sets of $X$ and $Y$ respectively. Then the image of $\mu$ under the map $f$ is denoted by $f(\mu)$ and is defined as $f(\mu)(y)=\left\{\begin{array}{c}\operatorname{Sup}\left\{\mu(x): x \in f^{-1}(\mathrm{y})\right\} ; \text { If } f^{-1}(\mathrm{y}) \neq \varnothing \\ 0 ; \quad \text { otherwise }\end{array}, \forall y \in Y\right.$. Also the preimage of $v$ under $f$ is denoted by $f^{-1}(v)$ and is defined as $f^{-1}(v)(x)=v(f(x)) ; \forall x \in \mathrm{X}$.

Remark (2.4) Note that $\mu(x) \leq f(\mu)(f(x)) ; \forall x \in \mathrm{X}$, and equality hold when $f$ is bijective.

Definition (2.5)[1] Let $\left(\mathrm{X}_{1}, \delta_{1}\right)$ and $\left(\mathrm{Y}_{2}, \delta_{2}\right)$ be FTSs. Then the function $f:\left(\mathrm{X}_{1}, \delta_{1}\right) \rightarrow\left(\mathrm{Y}_{2}, \delta_{2}\right)$ is (i) fuzzy continuous: if and only if for every $v \in \delta_{2} \Rightarrow f^{-1}(v) \in \delta_{1}$.

(ii) fuzzy open: if and only if for every $\mu \in \delta_{1} \Rightarrow f(\mu) \in \delta_{2}$.

(iii) fuzzy closed: if and only if image of every $\delta_{1}$-closed set is $\delta_{2}$-closed set.

(iv) fuzzy homeomorphism: if and only if $f$ is bijective, fuzzy continuous and fuzzy open (or fuzzy closed).

Definition (2.6)[10]Let $v$ be a fuzzy set in $X$ and $\delta$ is a fuzzy topology on $X$. Then the induced fuzzy topology on $v$ is the family of subsets of $v$ which are the intersections with $v$ of $\delta$-open fuzzy sets in X. The induced fuzzy topology is denoted by $\delta_{v}$ i.e., $\delta_{v}=\{\mu \cap v: \forall \mu \in \delta\}$, and the pair $\left(v, \delta_{v}\right)$ is called a fuzzy subspace of $(\mathrm{X}, \delta)$.

Definition(2.7)[10]For any $\lambda \in(0,1]$ and $x \in \mathrm{X}$, a fuzzy set $x_{\lambda}$ in $\mathrm{X}$ is called a fuzzy point in $\mathrm{X}$ if

$$
x_{\lambda}(y)=\left\{\begin{array}{cc}
\lambda & \text { if } \mathrm{y}=x \\
0 & \text { otherwise }
\end{array}, \forall \mathrm{y} \in \mathrm{X}\right.
$$

The fuzzy point $x_{\lambda}$ is said to belongs to the fuzzy set $\mu$, denoted by $x_{\lambda} \in \mu$, iff $\mu(x) \geq \lambda$. 
In particular, $x_{\lambda} \leq y_{\sigma} \Leftrightarrow x=y$ and $\lambda \leq \sigma$.

The characteristic mapping of a subset $\mathrm{A}$ of $\mathrm{X}$ is denoted by $\chi_{\mathrm{A}}$ and is defined as

$$
\chi_{A}(x)=\left\{\begin{array}{l}
1 \quad \text { if } x \in A \\
0 \text { otherwise }
\end{array}, \forall x \in \mathrm{X}\right.
$$

Obviously, a characteristic function $\chi_{\mathrm{A}}$ is also a fuzzy set on $\mathrm{X}$ and for any non-empty subsets $\mathrm{A}$ and $\mathrm{B}$ of $\mathrm{X}$, we have $\mathrm{A} \subseteq \mathrm{B}$ if and only if $\chi_{\mathrm{A}} \subseteq \chi_{\mathrm{B}}$.

Definition (2.8) [5] Let $\left(\mathrm{B},+,{ }^{*},{ }^{\prime}, 0_{\mathrm{B}}, 1_{\mathrm{B}}\right)$ be a Boolean algebra and $\mu \in \mathrm{I}^{\mathrm{B}}$ be a fuzzy set, then $\mu$ is called a fuzzy Boolean subalgebra (FBSA) of B if for all $x, y \in \mathrm{B}$

(i) $\quad \mu(x+y) \geq \min \{\mu(x), \mu(y)\}$;

(ii) $\quad \mu(x * y) \geq \min \{\mu(x), \mu(y)\}$;

(iii) $\quad \mu\left(x^{\prime}\right)=\mu(x)$.

Lemma (2.9) For any fuzzy Boolean subalgebra $\mu$ of Boolean algebra $\left(B,+,{ }^{*},{ }^{\prime}, 0_{\mathrm{B}}, 1_{\mathrm{B}}\right)$ the following holds

(i) $\quad \mu\left(1_{\mathrm{B}}\right) \geq \mu(x), \forall x \in \mathrm{B}$

(ii) $\quad \mu\left(0_{\mathrm{B}}\right) \geq \mu(x), \forall x \in \mathrm{B}$

(iii) $\quad \mu\left(0_{\mathrm{B}}\right)=\mu\left(1_{\mathrm{B}}\right)$

Proof. (i) Since $\mu\left(1_{\mathrm{B}}\right)=\mu\left(x+x^{\prime}\right) \geq \min \left\{\mu(x), \mu\left(x^{\prime}\right)\right\}=\min \{\mu(x), \mu(x)\}=\mu(x), \forall x \in \mathrm{B}$

(ii) Also, $\mu\left(0_{\mathrm{B}}\right)=\mu\left(x x^{\prime}\right) \geq \min \left\{\mu(x), \mu\left(x^{\prime}\right)\right\}=\min \{\mu(x), \mu(x)\}=\mu(x), \forall x \in \mathrm{B}$

(iii)From (i) and (ii), we get $\mu\left(1_{\mathrm{B}}\right) \geq \mu\left(0_{\mathrm{B}}\right)$ and $\mu\left(0_{\mathrm{B}}\right) \geq \mu\left(1_{\mathrm{B}}\right)$ and so $\mu\left(0_{\mathrm{B}}\right)=\mu\left(1_{\mathrm{B}}\right)$

Now, the following results are easy to verify

Result (2.10)(i) If $\mathrm{H}$ is a subset of a Boolean algebra $\mathrm{B}$, then $\chi_{\mathrm{H}}$ is a fuzzy Boolean subalgebra of $\mathrm{B}$ if and only if $\mathrm{H}$ is a Boolean subalgebra of $\mathrm{B}$.

(ii) All constant fuzzy sets of a Boolean algebra B are fuzzy Boolean subalgebra of B.

\section{Boolean Algebraic Fuzzy Topology}

In this section, we will introduce Boolean algebraic fuzzy topology and give basic properties of this structure. We also discuss similarities with and difference from other fuzzy topological spaces.

Definition (3.1) Let $\left(\mathrm{B},+,{ }^{*},{ }^{\prime}, 0_{\mathrm{B}}, 1_{\mathrm{B}}\right)$ be a Boolean algebra and $\mathrm{I}^{\mathrm{B}}$ denotes the set of all fuzzy subsets of $\mathrm{B}$. Then the collection $\mathrm{T}=\left\{\mu: \mu \in \mathrm{I}^{\mathrm{B}}\right.$ such that $\mu(x)=\mu\left(x^{\prime}\right)$ for all $\left.x \in \mathrm{B}\right\}$ is a fuzzy topology on $\mathrm{B}$ and $B$ with this topology is called Boolean algebraic fuzzy topological space (BAFTS). It is denoted by (B,T). Proof. Since $\varnothing_{\sim}$ and $\mathrm{B}_{\sim}$ are constant fuzzy sets on $\mathrm{B}$ such that $\varnothing_{\sim}(x)=\varnothing_{\sim}\left(x^{\prime}\right)=0$, and $\mathrm{B}_{\sim}(x)=\mathrm{B}_{\sim}\left(x^{\prime}\right)=1$, for all $x \in \mathrm{B}$. Therefore, $\varnothing_{\sim} \in \mathrm{T}$ and $\mathrm{B}_{\sim} \in \mathrm{T}$.

Next, let $\mu, v \in \mathrm{T}$ be any two members and $x \in \mathrm{B}$ be any element, then $(\mu \cap \nu)(x)=\min \{\mu(x), v(x)\}=\min \left\{\mu\left(x^{\prime}\right), v\left(x^{\prime}\right)\right\}=(\mu \cap \nu)\left(x^{\prime}\right)$. Thus $\mu \cap \nu \in \mathrm{T}$.

Further, let $\left\{\mu_{\mathrm{i}}: \mathrm{i} \in \Lambda\right\} \subseteq \mathrm{T}$.

Now, $\left(\bigcup_{i \in \Lambda} \mu_{i}\right)(x)=\operatorname{Sup}_{i \in \Lambda}\left\{\mu_{i}(x)\right\}=\operatorname{Sup}_{i \in \Lambda}\left\{\mu_{i}\left(x^{\prime}\right)\right\}=\left(\bigcup_{i \in \Lambda} \mu_{i}\right)\left(x^{\prime}\right)$. So that $\bigcup_{i \in \Lambda} \mu_{i} \in \mathrm{T}$.

Hence $\mathrm{T}$ is a fuzzy topology on $\mathrm{B}$.

Remark (3.2) A fuzzy set $\mu$ of a BAFTS (B,T) is called T- closed if $\mu^{\mathrm{c}} \in \mathrm{T}$. One can easily verify that $\varnothing_{\sim}$ and $\mathrm{B}_{\sim}$ are T-closed and if $\mu, v$ are T-closed, then $\mu \cup \nu$ is also T-closed and if $\left\{\mu_{\mathrm{i}}: \mathrm{i} \in \Lambda\right\}$ be an arbitrary family of T-closed sets, then $\bigcap_{i \in \Lambda} \mu_{i}$ is also T-closed set.

Theorem (3.3) A fuzzy set $\mu$ of the BAFTS B is T-closed if and only if $\mu$ is T-open.

Proof. Let $\mu$ be T-open set, then $\mu(x)=\mu\left(x^{\prime}\right)$ for all $x \in \mathrm{B}$.

Now, $\mu^{\mathrm{c}}(x)=1-\mu(x)=1-\mu\left(x^{\prime}\right)=\mu^{\mathrm{c}}\left(x^{\prime}\right)$. So $\mu^{\mathrm{c}}$ is T-open i.e., $\mu$ is T-closed. 
Converse is also true.

Now, we give an example of discrete FTS which is not a BAFTS.

Example (3.4) Let $\mathrm{B}=\left\{0_{\mathrm{B}}, a, b, 1_{\mathrm{B}}\right\}$ be the diamond Boolean algebra, where the three operations + , $*$ and $'$ are defined as

$$
x+y=\operatorname{Sup}\{x, y\} \text { and } x * y=\operatorname{Inf}\{x, y\} \text { such that } 0_{\mathrm{B}}^{\prime}=1_{\mathrm{B}}, 1_{\mathrm{B}}^{\prime}=0_{\mathrm{B}}, a^{\prime}=b \text { and } b^{\prime}=a \text {. }
$$

Let $\delta=\mathrm{I}^{\mathrm{B}}$ be the discrete fuzzy topology on $\mathrm{B}$. We show that $(\mathrm{B}, \delta)$ is not a BAFTS.

Let $\mathrm{H}=\left\{0_{\mathrm{B}}, a, 1_{\mathrm{B}}\right\}$ be a subset of $\mathrm{B}$. Define a fuzzy set $\mu$ on $\mathrm{B}$ as follows:

$$
\mu(x)=\left\{\begin{array}{l}
\lambda \quad \text { if } x \in H \\
0 \quad \text { if } x \in B \backslash H
\end{array} \text {, where } \lambda \in(0,1] .\right.
$$

Then $\mu(a)=\lambda$, but $\mu\left(a^{\prime}\right)=\mu(b)=0$ i.e., $\mu(a) \neq \mu\left(a^{\prime}\right)$, so $\mu \notin \mathrm{T}$, where T is the BAFT on B. Hence $\delta$ is not a BAFT on B.

Now, we show that under certain condition on B, a BAFTS is also a discrete FTS.

Theorem (3.5) Let $(\mathrm{B}, \mathrm{T})$ be a BAFTS such that $x^{\prime}=x, \forall x \in \mathrm{B}$. Then $(\mathrm{B}, \mathrm{T})$ is a discrete FTS.

Proof. Let $\mu \in \mathrm{I}^{\mathrm{B}}$ and $x \in \mathrm{B}$ be any element. Then $\mu(x)=\mu\left(x^{\prime}\right) \Rightarrow \mu \in \mathrm{T}$. So $\mathrm{I}^{\mathrm{B}} \subseteq \mathrm{T}$. Hence $\mathrm{T}$ is a discrete fuzzy topology on B.

Note (3.6) We notice that the Theorem (3.5) holds only in the case when the Boolean algebra B is trivial one. This implies that there does not exists non-trivial Boolean algebra B for which the BAFTS is a discrete fuzzy topological space.

Remark (3.7)(i) If $\mathrm{B}$ is a Boolean algebra such that there exist atleast one element $x$, such that $x \notin\left\{0_{\mathrm{B}}, 1_{\mathrm{B}},\right\}$, then the BAFTS on B is not a discrete FTS.

(ii) If $|\mathrm{B}| \geq 2$, then the BAFTS $(\mathrm{B}, \mathrm{T})$ is not an indiscrete FTS, i.e., there exist $\mu \in \mathrm{I}^{\mathrm{B}}$, which is different from $\varnothing_{\sim}$ and $\mathrm{B}_{\sim}$ such that $\mu \in \mathrm{T}$.

Theorem (3.8) Every fuzzy Boolean subalgebra of a Boolean algebraic fuzzy topological space B is Topen set.

Proof.Let $\kappa$ be fuzzy Boolean subalgebra of a Boolean algebra B. Then by Definition (2.8), we have $\kappa\left(x^{\prime}\right)=\kappa(x), \forall x \in \mathrm{B}$ i.e., $\kappa \in \mathrm{T}$. Hence $\kappa$ is T- open set.

Remark (3.9) Converse of Theorem (3.7) is not true i.e., T-open set does not need to be fuzzy Boolean subalgebra of B.

Example (3.10) Consider $\mathrm{B}=\left\{0_{\mathrm{B}}, a, b, 1_{\mathrm{B}}\right\}$ be the diamond Boolean algebra as defined in Example (3.4). Let $\mathrm{H}=\{a, b\}$ be a subset of $\mathrm{B}$. Define a fuzzy set $\mu$ on $\mathrm{B}$ as follows:

$\mu(x)=\left\{\begin{array}{l}\lambda \quad \text { if } x \in H \\ 0 \quad \text { if } x \in B \backslash H\end{array}\right.$, where $\lambda \in(0,1]$. Let $\mathrm{T}$ be the BAFT defined on $\mathrm{B}$.

Note that, $\mu \in \mathrm{T}$ however, $\mu$ is a not fuzzy Boolean subalgebra of $\mathrm{B}$, for $\mu(a+b)=\mu\left(1_{\mathrm{B}}\right)=0 \nsucceq \lambda=\min \{\mu(a), \mu(b)\}$.

Now, the question arises, when a T-open set in BAFTS $(\mathrm{B}, \mathrm{T})$ is a fuzzy Boolean subalgebra of B. In this direction, we first prove the following lemma

Lemma (3.11) Let $\mu$ be a fuzzy Boolean subalgebra of the Boolean algebra B, then $\forall x_{\alpha}, \mathrm{y}_{\beta} \in \mu$ we have $(x y)_{\gamma} \in \mu$ and $(x+\mathrm{y})_{\gamma} \in \mu$, where $\gamma=\min \{\alpha, \beta\}$

Proof. Let $\mu$ be a fuzzy Boolean subalgebra of B and $x_{\alpha}, \mathrm{y}_{\beta} \in \mu$ then, we have $\mu(x) \geq \alpha$ and $\mu(y) \geq \beta \quad \therefore \min \{\mu(x), \mu(y)\} \geq \min \{\alpha, \beta\}=\gamma$.

As $\mu$ is fuzzy Boolean subalgebra of B

$\therefore \mu(x y) \geq \min \{\mu(x), \mu(y)\} \geq \min \{\alpha, \beta\}=\gamma$ and $\mu(x+y) \geq \min \{\mu(x), \mu(y)\} \geq \min \{\alpha, \beta\}=\gamma$.

This implies that $(x y)_{\gamma} \in \mu$ and $(x+y)_{\gamma} \in \mu$. 
Theorem (3.12) Let $\mu$ be T-open set in BAFTS (B,T), then $\mu$ is a fuzzy Boolean subalgebra of B if and only if $\forall x_{\alpha}, \mathrm{y}_{\beta} \in \mu$, we have $(x y)_{\gamma} \in \mu$ and $(x+\mathrm{y})_{\gamma} \in \mu$, where $\gamma=\min \{\alpha, \beta\}$.

Proof. When $\mu \in \mathrm{T}$ and $\mu$ is a fuzzy Boolean subalgebra of $\mathrm{B}$, then the result follows by Lemma (3.11)

Conversely, let $\mu$ be $\mathrm{T}$-open set such that $\forall x_{\alpha}, \mathrm{y}_{\beta} \in \mu$, we have

$(x y)_{\gamma} \in \mu$ and $(x+y)_{\gamma} \in \mu$, where $\gamma=\min \{\alpha, \beta\}$.

To show that $\mu$ is a fuzzy Boolean subalgebra of B.

Let $x, y \in \mathrm{B}$ be any element and suppose that $\mu(x)=\alpha$ and $\mu(\mathrm{y})=\beta$.

Suppose that $\alpha \leq \beta$, then $\min \{\alpha, \beta\}=\alpha$.

Now, $\mu(x)=\alpha$ and $\mu(y)=\beta \geq \alpha$. Then $x_{\alpha}, y_{\alpha} \in \mu \Rightarrow(x y)_{\alpha} \in \mu$ and $(x+y)_{\alpha} \in \mu$

i.e., $\mu(x y) \geq \alpha=\min \{\alpha, \beta\}=\min \{\mu(x), \mu(y)\}$ and $\mu(x+y) \geq \alpha=\min \{\mu(x), \mu(y)\}$.

The case when $\beta \leq \alpha$ is similar. Thus in both the cases, we find that

$\mu(x y) \geq \min \{\mu(x), \mu(y)\}$ and $\mu(x+y) \geq \min \{\mu(x), \mu(y)\}$.

As $\mu \in \mathrm{T}$, therefore, $\mu(x)=\mu\left(x^{\prime}\right)$ for all $x \in \mathrm{B}$.

Hence $\mu$ is fuzzy Boolean subalgebra of B.

Theorem (3.13) Let $\mathrm{H}$ be a Boolean subalgebra of a BAFTS $(B, T)$, then the relative fuzzy topology $\mathrm{T}_{\mathrm{H}}$ on $\mathrm{H}$ is a BAFT on $\mathrm{H}$.

Proof. The subset $\mathrm{H}$ of B (in the ordinary sense) has a characteristic function $\chi_{\mathrm{H}}$ such that

$$
\chi_{H}(x)=\left\{\begin{array}{ll}
1 & \text { if } x \in \mathrm{H} \\
0 & \text { if } x \notin \mathrm{H}
\end{array} .\right.
$$

Clearly, $\chi_{\mathrm{H}}(x)=1, \forall x \in \mathrm{H}$ is a constant fuzzy set on $\mathrm{H}$.

Let $\mathrm{T}_{\mathrm{H}}=\left\{\mu \cap \chi_{\mathrm{H}}: \forall \mu \in \mathrm{T}\right\}$. Then we claim that $\mathrm{T}_{\mathrm{H}}$ is a BAFT on $\mathrm{H}$.

For, choose $\varnothing_{\sim} \in \mathrm{T}$, then as $\left(\varnothing_{\sim} \cap \chi_{\mathrm{H}}\right)(x)=\min \left\{\varnothing_{\sim}(x), \chi_{\mathrm{H}}(x)\right\}=\varnothing_{\sim}(x)$, so we have $\varnothing_{\sim} \in \mathrm{T}_{\mathrm{H}}$.

To show that $\mathrm{H}_{\sim} \in \mathrm{T}_{\mathrm{H}}$, choose $\chi_{\mathrm{B}}$ the characteristic function for $\mathrm{B}$, then

$$
\left(\chi_{\mathrm{B}} \cap \chi_{\mathrm{H}}\right)(x)=\min \left\{\chi_{\mathrm{B}}(x), \chi_{\mathrm{H}}(x)\right\}=\chi_{\mathrm{H}}(x) \text {, so we have } \mathrm{H}_{\sim} \in \mathrm{T}_{\mathrm{H}} \text {. }
$$

Further, let $\left\{\mu_{\mathrm{i}} \cap \chi_{\mathrm{H}}: \mathrm{i} \in \Lambda\right\} \subseteq \mathrm{T}_{\mathrm{H}}$. Then, $\bigcup_{i \in \Lambda}\left(\mu_{i} \cap \chi_{H}\right)=\left(\bigcup_{i \in \Lambda} \mu_{i}\right) \cap \chi_{H} \in T_{H}$.

Also, let $\mu_{1} \cap \chi_{\mathrm{H}}$ and $\mu_{2} \cap \chi_{\mathrm{H}}$ be any two members of $\mathrm{T}_{\mathrm{H}}$. Then $\left(\mu_{1} \cap \chi_{\mathrm{H}}\right) \cap\left(\mu_{2} \cap \chi_{\mathrm{H}}\right)=\left(\mu_{1} \cap \mu_{2}\right) \cap \chi_{\mathrm{H}} \in \mathrm{T}_{\mathrm{H}}$. Hence $\mathrm{T}_{\mathrm{H}}$ is a fuzzy topology on $\mathrm{H}$.

Further, let $\mathrm{T}_{1}$ is a BAFT on $\mathrm{H}$, then we show that $\mathrm{T}_{1}=\mathrm{T}_{\mathrm{H}}$.

Let $\mu \cap \chi_{\mathrm{H}} \in \mathrm{T}_{\mathrm{H}}$, then $\left(\mu \cap \chi_{\mathrm{H}}\right)(x)=\min \left\{\mu(x), \chi_{\mathrm{H}}(x)\right\}=\min \left\{\mu\left(x^{\prime}\right), \chi_{\mathrm{H}}\left(x^{\prime}\right)\right\}=\left(\mu \cap \chi_{\mathrm{H}}\right)\left(x^{\prime}\right)$.

So, $\mu \cap \chi_{\mathrm{H}} \in \mathrm{T}_{1}$, i.e., $\mathrm{T}_{\mathrm{H}} \subseteq \mathrm{T}_{1}$.

Further, let $v \in \mathrm{T}_{1}$ and $\operatorname{so} v(x)=v\left(x^{\prime}\right), \forall x \in \mathrm{B}$.

Also, $v=v \cap \chi_{\mathrm{H}} \in \mathrm{T}_{\mathrm{H}}$. Therefore, $\mathrm{T}_{1} \subseteq \mathrm{T}_{\mathrm{H}}$ and so $\mathrm{T}_{1}=\mathrm{T}_{\mathrm{H}}$.

Hence relative fuzzy topology on $\mathrm{H}$ is the BAFT on $\mathrm{H}$.

\section{Functions on Boolean Algebraic Fuzzy Topological Spaces}

In this section, we study the behaviour of Boolean algebraic fuzzy topology under different Boolean homomorphism (isomorphism) and also under fuzzy continuity from one Boolean algebraic fuzzy topological space to another Boolean algebraic fuzzy topological space.

Theorem (4.1) Let $f: \mathrm{B}_{1} \rightarrow \mathrm{B}_{2}$ be a Boolean isomorphism and $\mathrm{T}_{1}, \mathrm{~T}_{2}$ be BAFTs on Boolean algebra $\mathrm{B}_{1}$ and $\mathrm{B}_{2}$ respectively. Then $f$ is a fuzzy homeomorphism between BAFTS $\left(\mathrm{B}_{1}, \mathrm{~T}_{1}\right)$ and $\left(\mathrm{B}_{2}, \mathrm{~T}_{2}\right)$.

Proof. Let $f: \mathbf{B}_{1} \rightarrow \mathbf{B}_{2}$ be a Boolean isomorphism, where $\mathbf{B}_{1}$ and $\mathbf{B}_{2}$ are Boolean algebras. We show that $f$ is a fuzzy homeomorphism. For this, we need to show that $f$ is a fuzzy continuous and fuzzy open map. For fuzzy continuity: let $v$ be any $\mathrm{T}_{2^{-}}$open set and $x \in \mathrm{B}_{1}$ be any element. Then 
$\left(f^{-1}(v)\right)(x)=v(f(x))=v\left[\{f(x)\}^{\prime}\right]=v\left(f\left(x^{\prime}\right)\right)=\left(f^{-1}(v)\right)\left(x^{\prime}\right)$. So $f^{-1}(v)$ is $\mathrm{T}_{1}$-open set. Hence $f$ is fuzzy continuous.

For fuzzy open mapping: let $\mu$ be any $\mathrm{T}_{1}$-open set and let $y \in \mathrm{B}_{2}$ be any element, then $\exists$ unique $x \in \mathrm{B}_{1}$ such that $f(x)=y$.

Now, $f(\mu)(y)=f(\mu)(f(x))=\mu(x)=\mu\left(x^{\prime}\right)=f(\mu)\left(f\left(x^{\prime}\right)\right)=f(\mu)\left(f(x)^{\prime}\right)=f(\mu)\left(y^{\prime}\right)$.

i.e., $f(\mu) \in \mathrm{T}_{2}$. Therefore, $f$ is fuzzy open mapping. Hence $f$ is a fuzzy homeomorphism.

Theorem (4.2) Let $f:(\mathrm{B}, \mathrm{T}) \rightarrow(\mathrm{B}, \mathrm{T})$ be a mapping from a BAFTS $(\mathrm{B}, \mathrm{T})$ onto itself defined by $f(x)=x^{\prime}$; $\forall \quad x \in \mathrm{B}$. Then $f$ is bijective and fuzzy continuous.

Proof. Clearly, $f$ is one-one and onto. Let $v \in \mathrm{T}$ be any open set. Then we show that $f^{-1}(v) \in \mathrm{T}$.

Let $x \in \mathrm{B}$ be any element $\Rightarrow f(x)=x^{\prime}$.

Now, $f^{-1}(v)(x)=v(f(x))=v\left(x^{\prime}\right)=v(x)=v\left(f\left(x^{\prime}\right)\right)=f^{-1}(v)\left(x^{\prime}\right)$

So, $f^{-1}(v) \in \mathrm{T}$. Hence $f$ is fuzzy continuous.

Theorem (4.3) If $f:\left(\mathrm{B}_{1}, \mathrm{~T}_{1}\right) \rightarrow\left(\mathrm{B}_{2}, \mathrm{~T}_{2}\right)$ be a mapping defined by $f\left(x^{\prime}\right)=(f(x))^{\prime}, \forall x \in \mathrm{B}_{1}$. Then $f$ is fuzzy continuous.

Proof. Let $x \in \mathrm{B}_{1}$ be any element. Let $v \in \mathrm{T}_{2}$ be any open set. Then we show that $f^{-1}(v) \in \mathrm{T}_{1}$.

Now, $f^{-1}(v)(x)=v(f(x))=v\left((f(x))^{\prime}\right)=v\left(f\left(x^{\prime}\right)\right)=f^{-1}(v)\left(x^{\prime}\right) \quad[$ As $v \in \mathrm{T}$ ]

So, $f^{-1}(v) \in \mathrm{T}$. Hence $f$ is fuzzy continuous.

Theorem (4.4) Let the mapping $f:\left(\mathrm{B}_{1}, \mathrm{~T}_{1}\right) \rightarrow\left(\mathrm{B}_{2}, \mathrm{~T}_{2}\right)$ be fuzzy continuous. Then

$$
f\left(x^{\prime}\right)=(f(x))^{\prime} \text { or }(f(x)), \forall x \in \mathrm{B}_{1} \text {. }
$$

Proof. As $f$ is continuous. Therefore, if $v$ be any $\mathrm{T}_{2}$ - open set, then $f^{-1}(v)$ is $\mathrm{T}_{1}$-open set.

Let $x \in \mathrm{B}_{1}$ be any element, then we have $f^{-1}(v)(x)=f^{-1}(v)\left(x^{\prime}\right) \Rightarrow v(f(x))=v\left(f\left(x^{\prime}\right)\right)$.

Let $\mathrm{H}=\left\{f(x),(f(x))^{\prime}\right\}$. Clearly, $\mathrm{H} \subseteq \mathrm{B}_{2}$. Define the fuzzy set $\theta$ on $\mathrm{B}_{2}$ as follow:

$$
\theta(y)=\left\{\begin{array}{ll}
\lambda & \text { if } \mathrm{y} \in H \\
0 & \text { if } \mathrm{y} \notin H
\end{array}, \text { where } \lambda \in(0,1] .\right.
$$

Obviously, $\theta \in \mathrm{T}_{2}$. As proved earlier, $\theta(f(x))=\theta\left(f\left(x^{\prime}\right)\right)$. This means that either $f\left(x^{\prime}\right)=(f(x))^{\prime}$ or $(f(x))$. Hence proved.

Note (4.5) If in Theorem (4.4) $f$ is one-one or $f\left(x^{\prime}\right) \neq(f(x))^{\prime}$ for all $x \in \mathrm{B}_{1}$, then we have the following theorem:

Theorem (4.6) Let $f:\left(\mathrm{B}_{1}, \mathrm{~T}_{1}\right) \rightarrow\left(\mathrm{B}_{2}, \mathrm{~T}_{2}\right)$ be a bijective mapping. Then $f$ is fuzzy continuous if and only if $f\left(x^{\prime}\right)=(f(x))^{\prime}, \forall x \in \mathrm{B}_{1}$.

Theorem (4.7) Boolean algebraic fuzzy topological space is a fuzzy topological property.

Proof. Let $\left(\mathrm{B},+, \bullet,{ }^{\prime}, 0_{\mathrm{B}}, 1_{\mathrm{B}}\right)$ be a Boolean algebra and $(\mathrm{B}, \mathrm{T})$ be a BAFTS. Suppose that $f:(\mathrm{B}, \mathrm{T})$

$\rightarrow\left(\mathrm{X}, \mathrm{T}^{\prime}\right)$ be a fuzzy homeomorphism. We will show that $\left(\mathrm{X}, \mathrm{T}^{\prime}\right)$ is also a BAFTS. To prove this, we first show that $\mathrm{X}$ is a Boolean algebra under the operation:

For any $\mathrm{y}_{\mathrm{i}} \in \mathrm{X} \exists$ 's unique $x_{\mathrm{i}} \in \mathrm{B}$ such that $f\left(x_{\mathrm{i}}\right)=\mathrm{y}_{\mathrm{i}}$, where $\mathrm{i}=1,2,3$. We have

$\mathrm{y}_{1} \otimes \mathrm{y}_{2}=f\left(x_{1}\right) \otimes f\left(x_{2}\right)=f\left(x_{1} \bullet x_{2}\right)$ i.e., $\mathrm{y}_{1 \otimes \mathrm{y}_{2}}=f\left(f^{-1}\left(\mathrm{y}_{1}\right) \bullet f^{-1}\left(y_{2}\right)\right)=f\left(x_{1} \bullet x_{2}\right)$ and

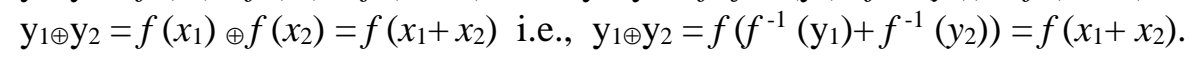

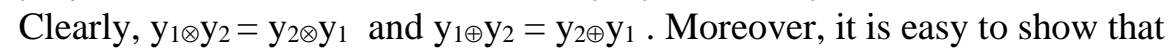

$\mathrm{y}_{1} \otimes\left(\mathrm{y}_{2} \otimes \mathrm{y}_{3}\right)=\left(\mathrm{y}_{1} \otimes \mathrm{y}_{2}\right)_{\otimes} \mathrm{y}_{3}$ and $\mathrm{y}_{1 \oplus}\left(\mathrm{y}_{2} \oplus \mathrm{y}_{3}\right)=\left(\mathrm{y}_{1} \oplus \mathrm{y}_{2}\right)_{\oplus} \mathrm{y}_{3}$.

Also, $\mathrm{y}_{1 \otimes}\left(\mathrm{y}_{2} \oplus \mathrm{y}_{3}\right)=\mathrm{y}_{1 \otimes}\left[f\left(x_{2}+x_{3}\right)\right]=f\left(x_{1}\right) \otimes\left[f\left(x_{2}+x_{3}\right)\right]=f\left\{x_{1} \bullet\left(x_{2}+x_{3}\right)\right\}=f\left\{x_{1} \bullet x_{2}+x_{1} \bullet x_{3}\right\}$

$=f\left(x_{1} \bullet x_{2}\right) \oplus f\left(x_{1} \bullet x_{3}\right)=\mathrm{y}_{1} \otimes \mathrm{y}_{2} \oplus \mathrm{y}_{1} \otimes \mathrm{y}_{3}$.

Similarly, we can show that $\mathrm{y}_{1 \oplus}\left(\mathrm{y}_{2} \otimes \mathrm{y}_{3}\right)=\left(\mathrm{y}_{1 \oplus} \mathrm{y}_{2}\right) \otimes\left(\mathrm{y}_{1 \oplus} \mathrm{y}_{3}\right)$

There exists elements $f\left(1_{\mathrm{B}}\right)=1_{\mathrm{X}}$ and $f\left(0_{\mathrm{B}}\right)=0_{\mathrm{X}}$ in $\mathrm{X}$ such that $\mathrm{y}{ }_{\otimes} 1_{\mathrm{X}}=\mathrm{y}$ and $\mathrm{y}_{\oplus} 0_{\mathrm{X}}=\mathrm{y}, \forall \mathrm{y} \in \mathrm{X}$

Existence of inverse: let $\mathrm{y} \in \mathrm{X}$. Then $\exists x \in \mathrm{B}$ such that $\mathrm{y}=f(x)$. We show that $\mathrm{y}^{\prime}=f\left(x^{\prime}\right)$ is the complement of $y$.

Now, $\mathrm{y} \otimes_{\otimes} \mathrm{y}^{\prime}=f(x)_{\otimes} f\left(x^{\prime}\right)=f\left(x \bullet x^{\prime}\right)=f\left(1_{\mathrm{B}}\right)=1_{\mathrm{X}}$ and $\mathrm{y}_{\oplus} \mathrm{y}^{\prime}=f(x)_{\oplus} f\left(x^{\prime}\right)=f\left(x+x^{\prime}\right)=f\left(0_{\mathrm{B}}\right)=0_{\mathrm{X}}$.

Hence $\mathrm{X}$ is a Boolean algebra under the operations $\oplus, \otimes$ and '. 
Next, we show that the topology $\mathrm{T}^{\prime}$ is the BAFT on $\mathrm{X}$.

Let $\mathrm{T}^{\prime \prime}$ be the BAFT on X. Then we show that $\mathrm{T}^{\prime}=\mathrm{T}^{\prime \prime}$.

Let $\mathrm{y} \in \mathrm{X}$ be any element then $\exists$ a unique $x \in \mathrm{B}$ such that $f(x)=\mathrm{y}$.

Let $v \in T^{\prime}$. As $f$ is continuous $\therefore f^{-1}(v) \in \mathrm{T}$. Also, because T is a BAFT on B

$\therefore f^{-1}(v)(x)=f^{-1}(v)\left(x^{\prime}\right) ; \quad \forall x \in \mathrm{B}$ i.e., $v(f(x))=v\left(f\left(x^{\prime}\right)\right)=v\left((f(x))^{\prime}\right)$

i.e., $\quad v(\mathrm{y})=v\left(\mathrm{y}^{\prime}\right) ; \forall \mathrm{y} \in \mathrm{X} \Rightarrow v \in \mathrm{T}^{\prime \prime} \quad\left[\because \mathrm{T}^{\prime \prime}\right.$ is a BAFT on $\left.\mathrm{X}\right]$.

Thus $v \in T^{\prime} \Rightarrow v \in \mathrm{T}^{\prime \prime} \quad \therefore \quad \mathrm{T}^{\prime} \subseteq \mathrm{T}^{\prime \prime}$.

Conversely, let $v \in \mathrm{T}^{\prime \prime}$. Then for any element $\mathrm{y} \in \mathrm{X}, \exists$ unique $x \in \mathrm{B}$ such that $f(x)=\mathrm{y}$.

Now, $\quad v(y)=v\left(y^{\prime}\right) \Rightarrow v(f(x))=v\left((f(x))^{\prime}\right)=v\left(f\left(x^{\prime}\right)\right)$

i.e., $\quad f^{-1}(v)(x)=f^{-1}(v)\left(x^{\prime}\right) \Rightarrow f^{-1}(v) \in T$.

As $f$ is fuzzy open mapping $\therefore f\left(f^{-1}(v)\right) \in T^{\prime} \quad$ i.e. $\quad v \in \mathrm{T}^{\prime}$.

Thus $v \in \mathrm{T}^{\prime \prime} \Rightarrow v \in \mathrm{T}^{\prime} \quad$ so $\quad \mathrm{T}^{\prime \prime} \subseteq \mathrm{T}^{\prime}$. Hence $\mathrm{T}^{\prime}=\mathrm{T}^{\prime \prime}$.

Corollary (4.8)Let $f:\left(\mathrm{B}_{1}, \mathrm{~T}_{1}\right) \rightarrow\left(\mathrm{B}_{2}, \mathrm{~T}_{2}\right)$ be fuzzy continuous mapping and $\mathrm{K}=\left\{x \in \mathrm{B}_{1}: f(x)=0_{\mathrm{B} 2}\right.$ and $f$ $\left.\left(x^{\prime}\right)=1_{\mathrm{B} 2}\right\}$. Then the topology induced on the set $\mathrm{K}$ is a BAFT.

Proof. Let $\mathrm{T}_{\mathrm{K}}=\left\{\mu \cap \chi_{\mathrm{K}}: \forall \mu \in \mathrm{T}_{1}\right\}$. Then we claim that $\mathrm{T}_{\mathrm{K}}$ is a BAFT on $\mathrm{K}$.

Let $x \in \mathrm{K}$ be any element, then $f(x)=0_{\mathrm{B} 2}$ and $f\left(x^{\prime}\right)=1_{\mathrm{B} 2}$.

Let $v \in \mathrm{T}_{2}$ be any open set in $\mathrm{B}_{2}$. As $f$ is continuous $\therefore f^{-1}(v) \in \mathrm{T}_{1}$.

Now, $\left(f^{-1}(v) \cap \chi_{\mathrm{K}}\right)(x)=\min \left\{f^{-1}(v)(x), \chi_{\mathrm{K}}(x)\right\}$

$$
\begin{aligned}
& =\min \left\{v(f(x)), \chi_{\mathrm{K}}(x)\right\} \\
& =\min \left\{v\left(0_{\mathrm{B} 2}\right), \chi_{\mathrm{K}}(x)\right\} \quad\left[\text { As } \chi_{\mathrm{K}}(x)=1\right] \\
& =v\left(0_{\mathrm{B} 2}\right)=v\left(1_{\mathrm{B} 2}\right)=v\left(f\left(x^{\prime}\right)\right) \\
& =\min \left\{v\left(f\left(x^{\prime}\right)\right), \chi_{\mathrm{K}}\left(x^{\prime}\right)\right\} \quad\left[\text { As } \chi_{\mathrm{K}}\left(x^{\prime}\right)=1\right] \\
& =\min \left\{f^{-1}(v)\left(x^{\prime}\right), \chi_{\mathrm{K}}\left(x^{\prime}\right)\right\} \\
& =\left(f^{-1}(v) \cap \chi_{\mathrm{K}}\right)\left(x^{\prime}\right)
\end{aligned}
$$

Hence $T_{K}$ is a BAFT on $K$.

\section{Product of Boolean Algebraic Fuzzy Topological Spaces}

In this section, we study the product of Boolean algebraic fuzzy topological spaces. We also discuss similarities with difference from product of fuzzy topological spaces

Theorem (5.1)Let $\left(B_{1}, T_{1}\right)$ and $\left(B_{2}, T_{2}\right)$ be two BAFTS. Then product fuzzy topology on $B_{1} \times B_{2}$ is contained in the BAFT on $\mathrm{B}_{1} \times \mathrm{B}_{2}$. The equality between the two topologies does not need to be hold.

Proof. We know that $\mathrm{B}_{1} \times \mathrm{B}_{2}$ is a Boolean algebra under the operation defined by $\left(x_{1}, y_{1}\right) \otimes\left(x_{2}, y_{2}\right)=\left(x_{1} \bullet x_{2}, y_{1} \bullet y_{2}\right) ;\left(x_{1}, y_{1}\right) \oplus\left(x_{2}, y_{2}\right)=\left(x_{1}+x_{2}, y_{1}+y_{2}\right) ; \forall\left(x_{1}, y_{1}\right),\left(x_{2}, y_{2}\right) \in \mathrm{B}_{1} \times \mathrm{B}_{2}$ and $(x, y)^{\prime}=\left(x^{\prime}, y^{\prime}\right), \forall(x, y) \in \mathrm{B}_{1} \times \mathrm{B}_{2}$.

The element $\left(0_{1}, 0_{2}\right)$ and $\left(1_{1}, 1_{2}\right)$ be the lower bound and upper bound element of $\mathrm{B}_{1} \times \mathrm{B}_{2}$. Let $(x, y)$ be any element of $\mathrm{B}_{1} \times \mathrm{B}_{2}$. Then $\left(x^{\prime}, y^{\prime}\right)$ is the complement of $(x, y)$ in $\mathrm{B}_{1} \times \mathrm{B}_{2}$. Let $\mathrm{T}^{\prime}$ be the product fuzzy topology on $\mathrm{B}_{1} \times \mathrm{B}_{2}$ and $\mathrm{T}$ be the BAFT on $\mathrm{B}_{1} \times \mathrm{B}_{2}$.

We show that $\mathrm{T}^{\prime} \subseteq \mathrm{T}$. Let $\theta \in \mathrm{T}^{\prime}$ and let $\mathrm{z}=(x, y)$ be any element of $\mathrm{B}_{1} \times \mathrm{B}_{2}$. Then $\theta(\mathrm{z})=\theta(x, y)=(\mu \times v)(x, y)=\min \{\mu(x), v(\mathrm{y})\}=\min \left\{\mu\left(x^{\prime}\right), v\left(\mathrm{y}^{\prime}\right)\right\}=(\mu \times v)(x, y)^{\prime}=\theta(x, y)^{\prime}=\theta\left(\mathrm{z}^{\prime}\right)$ so that $\theta \in \mathrm{T}$. Hence $\mathrm{T}^{\prime} \subseteq \mathrm{T}$.

Next, we show that the equality between $\mathrm{T}$ and $\mathrm{T}^{\prime}$ does not need to be hold.

Let $\mathrm{B}_{1}=\left(\mathrm{D}_{6},+,,^{\prime}\right)$ and $\mathrm{B}_{2}=\left(\mathrm{D}_{30},+,,^{\prime}\right)$. Let $\mathrm{T}_{1}, \mathrm{~T}_{2}$ be the BAFT on $\mathrm{B}_{1}$ and $\mathrm{B}_{2}$ respectively. Further let $\mathrm{T}^{\prime}$ be the product fuzzy topology on $\mathrm{B}_{1} \times \mathrm{B}_{2}$ and $\mathrm{T}$ be the BAFT on $\mathrm{B}_{1} \times \mathrm{B}_{2}$. 
Consider the subset $\mathrm{H}=\{(2,6),(3,5)\}$ of $\mathrm{B}_{1} \times \mathrm{B}_{2}$. Define the fuzzy set $\eta$ on $\mathrm{B}_{1} \times \mathrm{B}_{2}$ such that $\eta((x, y))=\lambda$ if $(x, y) \in \mathrm{H}$ and 0 otherwise, where $\lambda \in(0,1]$.

Clearly, $\eta(x, y)^{\prime}=\eta(x, y) ; \forall(x, y) \in \mathrm{B}_{1} \times \mathrm{B}_{2}$ and so, $\eta \in \mathrm{T}$. But the smallest $\mathrm{T}^{\prime}$-open set containing $\eta$ is $\mu$ $\times v$, where $\mu \in \mathrm{T}_{1}$ and $v \in \mathrm{T}_{2}$ defined as:

$$
\mu(x)=\left\{\begin{array}{l}
\lambda \quad \text { if } x=2,3 \\
0 \quad ; \text { otherwise }
\end{array} \text { and } \quad v(x)=\left\{\begin{array}{l}
\lambda ; \text { if } x=6,5 \\
0 ; \text { otherwise }
\end{array}\right. \text {. Then }\right.
$$

$(\mu \times v)(x, y)=\min \{\mu(x), v(y)\}=\lambda ;$ if $(x, y) \in\{(2,6),(2,5),(3,6),(3,5)\}=\{2,3\} \times\{6,5\}$

and $(\mu \times v)(x, y)=0$; if $(x, y) \notin\{2,3\} \times\{6,5\}$.

Thus, $\eta \notin \mathrm{T}^{\prime}$. Hence, $\mathrm{T}^{\prime} \neq \mathrm{T}$.

Remark (5.2) In the above theorem (5.1), if we have $\mathrm{B}_{1}=\mathrm{B}_{2}=\mathrm{B}$ (say), even then the product fuzzy topology on B $\times$ B is not same as the BAFT on B $\times$ B.

\section{Conclusion}

In this paper, the notion of Boolean algebraic fuzzy topological spaces is introduced. It has been observed that the Boolean algebraic fuzzy topology is different from discrete and indiscrete fuzzy topology, the cases when they behave same have been examined. It is seen that Boolean algebraic fuzzy topology is a hereditary property subject to the Boolean subalgebra of the Boolean algebra B. Also it is noticed that Boolean algebraic fuzzy topology is a topological and productive property.

\section{Scope of Further Study}

Many properties like fuzzy connectedness, fuzzy compactness, fuzzy separation axioms, convergence of sequence in Boolean algebraic fuzzy topological space $(B, T)$ are yet to be examined. This work is under progress.

\section{Acknowledgement}

The author is highly thankful to the referee for his valuable suggestions for improving the quality of the paper.

\section{References}

[1] C. L. Chang, “Fuzzy Topological Spaces”, J. Math. Anal. Appl., 24 (1968), 182-190.

[2] R. Lowen, "Fuzzy Topological Spaces and Fuzzy Compactness", J. Math. Anal. Appl., 56 (1976), 621-633.

[3] A. Parvathi, K.N. Meenakshi, “Fuzzy Topological Boolean Algebras”, Indian J. pure appl. Math. 28(12), (1997), 1639-1648.

[4] A. Rosenfeld, "Fuzzy Groups", J. Math. Anal. Appl., 35 (1971), 512-517.

[5] Shaoquan Sun, "Fuzzy subalgebras and Fuzzy ideals of Boolean algebra", Fuzzy Systems and Mathematics, vol. 20(1) (2006) pp. 90-94.

[6] Shaoquan Sun, "Generalized Fuzzy Subalgebras of Boolean Algebras", Proceedings of the 2009 International Workshop on Information Security and Application (IWISA 2009) Qingdao, China, November 21-22, (2009).

[7] C. K. Wong, "Fuzzy Topology, Products and Quotient Theorems”, J. Math. Anal. Appl., 45 (1974), $512-521$. 
[8] L. A. Zadeh, "Fuzzy sets, Information and Control", 8 (1965), 338-353.

[9] Elliott Mendelson, "Schaum's outline of Boolean Algebra and Switching Circuits", McGraw Hill, (1970), ISBN 07-041460-2.

[10] N. Palaniappan, "Fuzzy Topology", $2^{\text {nd }}$ Edition Narosa Publishing House, India. 